\section{Long-term Mortality Outcomes of Hyperkalemic Patients in the Emergency Department Setting: A Case Control Study}

\section{Abstract}

Background: The impact of an episode of hyperkalemia on long term mortality is not well studied. Such a study is vital for development of a utilization strategy for new drugs for the treatment of hyperkalemia. To study the long term impact of hyperkalemia on mortality, we conducted a retrospective, observational casecontrol study in a tertiary care emergency department. We compared cases of non-hemolyzed serum potassium $>5.3 \mathrm{mEq} / \mathrm{L}$ with normokalemic controls. Allcause mortality and causes of death were determined from social security death index, national death index, and medical record review over a follow-up period of 490 patient years.

Methods and Findings: A total of 287 consecutive subjects ( 223 cases, 64 controls) were identified among 14,483 patients. Cox proportional hazard regression showed that for each $1 \mathrm{mEq} / \mathrm{L}$ increase in admit potassium, all-cause mortality Hazard Ratio was 1.54, 95\% Confidence Interval: 1.22-1.96. Subgroup analyses showed higher mortality in acute kidney injury and heart failure patients.

Conclusion: Hyperkalemia predicted all-cause mortality, when adjusted for age, sex, Charlson comorbidity index, and significant covariates (heart failure and end stage renal disease). The deleterious effect of admit hyperkalemia persisted in survivors from index episode throughout the study period. Cardiovascular mortality was statistically more prevalent. Future studies are needed for risk stratification strategies and mortality impact of newer drugs.

Keywords: Hyperkalemia; Mortality

Received: April 19, 2017; Accepted: April 22, 2017; Published: April 29, 2017

\author{
Venu Velagapudi', \\ John C O'Horo ${ }^{3}$, \\ Vladimir Glinskiii, \\ Mazen Al-Quadi ${ }^{4}$, \\ Philimon Gona ${ }^{2}$, \\ Aimee R Kroll-Desrosiers', \\ Anu Vellanki' and \\ Jeffrey S Stoff ${ }^{1}$
}

1 Department of Medicine, Renal Medicine Division, University of Massachusetts Medical School, Worcester, USA

2 Department of Quantitative Health Sciences, Division of Biostatistics and Health Services Research; University of Massachusetts Medical School, Worcester, USA

3 Department of Medicine, Division of Infectious Diseases, Mayo Clinic, Rochester, USA

4 Department of Medicine, Division of Pulmonary Critical Care and Sleep Medicine, Yale New Haven Hospital, New Haven, USA

Corresponding author: Venu Velagapudi

झ velagapv@gmail.com

Department of Medicine, Renal Medicine Division, University of Massachusetts Medical School, Worcester, USA.

Tel: 5089715236

Fax: 5088563111

Citation: Velagapudi V, O'Horo JC, Glinskii $\mathrm{V}$, et al. Long-term Mortality Outcomes of Hyperkalemic Patients in the Emergency Department Setting: A Case Control Study. Arch Med. 2017, 9:2.

Unfortunately, there is a knowledge gap in current literature on the long term effect of hyperkalemia of mortality especially in a generally representative population such as emergency department subjects. A study on the long term morality impact 
of hyperkalemia is vital for evolution of utilization strategies for new drugs; especially patriromer which showed longer term decrease in potassium levels over 12 weeks [6]. The paucity of existing data on long term mortality in hyperkalemia precludes the accurate future assessment of mortality benefits of these new drugs. Most published literature focuses on the effects of hyperkalemia on short-term mortality $[1,7]$. There is a paucity of published literature on the outcomes of hyperkalemia in survivors from the index episode.

Our study aims to address the knowledge gap on longer term mortality effects of hyperkalemia.

We conducted a retrospective, case-control study of hyperkalemic patients who presented to a tertiary care emergency department (ED). The principal aim was to study the predictors of both 1-year and long term all-cause mortality in hyperkalemia. The secondary aim was to study the causes of death in hyperkalemia with a focus on cardiovascular mortality.

\section{Methods}

\section{Study sample}

We examined a retrospective, observational, unmatched case-control design to study ED patients with hyperkalemia (initial serum $\mathrm{K}^{+}$value $>5.3 \mathrm{mEq} / \mathrm{L}$ ). The cases were comprised of consecutive ED patients in the University of Massachusetts medical system from January 1, 2009 through March 31, 2009 with true hyperkalemia. Controls were identified from the same setting of ED patients, but were ultimately found to have pseudohyperkalemia (hemolyzed blood samples) and were demonstrated to be normokalemic. Patients were excluded when hemolysis could not be reliably excluded (Appendix I).

The study was approved by the Institutional Review Board at the University of Massachusetts Medical School.

\section{Outcomes}

The primary endpoint was all-cause mortality determined by searching Social Security Death Index (SSDI) [8], national death index (NDI), and review of medical records. SSDI data was updated through April 2012. Patients not found in SSDI data were considered alive through January 1, 2012. If there was a conflict, the later date of either SSDI or manual chart review was used as the censoring date in follow-up time calculation. The causes of death were coded per ICD 9 and 10 were obtained from NDI, and manual review of medical records. We analyzed causes of death as determined by international classification of disease (ICD) codes in NDI database or chart review. We postulated that hyperkalemia contributes to arrhythmic or cardiovascular causes of death, and thus categorized patients as having a cardiac versus non-cardiac cause of death. The definition used for cardiac cause of death was ICD 10 codes 100 to I09, I11, I20 to I51, Q20 to Q24, and R95 to R99) [9] or ICD-9-CM codes 410-414 [10].

\section{Variables}

Raw and standardized potassium, as well as a three-level ordinal variable created using cut-points of $<5.3,5.3-6.0$, and $>6.0$
$(\mathrm{mEq} / \mathrm{L})$ were analyzed. A four-level ordinal variable for eGFR was also created using the following cut-points: <14.99, 15.00-29.99, 30.00-59.99 and $>60.00\left(\mathrm{ml} / \mathrm{min} / 1.73 \mathrm{~m}^{2}\right)$. Data on past medical history, medications, treatment, repeat admissions/emergency room visits, follow-up potassium, and creatinine/eGFR were obtained via manual chart review. eGFR was computed per isotope dilution mass spectrometry traceable-modified diet in renal disease (IDMS-MDRD) [11] (Equation 1 Appendix).

Charlson comorbidity index (CCl) [12], validated in ESRD, $\mathrm{HF}$, critical care and cancer patients [13], was used to evaluate the chronic disease burden of subjects. $\mathrm{CCl}$ can be used to calculate the likelihood of 1-year mortality; diagnostic performance of the $\mathrm{CCl}$ was compared against our hyperkalemia model. The AKI subgroup was constituted of those meeting RIFLE criteria [14] (50\% reduction in baseline eGFR). Severe AKI was defined as the need for initiation of hemodialysis or continuous renal replacement therapies.

\section{Statistical methods}

All covariates were assessed at initial ED evaluation. Baseline characteristics of patients were compared based on case or control status and $\mathrm{K}^{+}$levels at admission using standard statistical methods (Appendix III). Kaplan-Meier curves and log-ranks were used to compare survival times stratified by case control status, $\mathrm{K}^{+}$level, MDRD, and HF status.

Age and sex adjusted cumulative mortality rates were calculated for both cases and controls. After confirming the veracity of the proportionality hazards assumption, we used sex-pooled multivariable Cox proportional hazards regression models to relate concentrations of $\mathrm{K}^{+}$at admission to mortality [15]. C-statistic was used to examine the predictive utility of the Cox models [16].

To assess for any potential nonlinearity of relations across values of potassium concentrations at admission, we used panelized cubic splines adjusting for age and sex [17-19]. Further analysis of renal function was conducted by stratifying subjects into ESRD and $A K I$ subsets since admit GFR does not reflect dynamic changes in renal function. We studied a severe AKI subset defined as need for initiation of hemodialysis or continuous renal replacement therapy. All data analysis was performed using SAS software, Version 9.2 (SAS Institute Inc., Cary, NC, USA).

\section{Results}

A total of 14,483 patients presented to the ED during the study period. Evaluation of these consecutive episodes yielded 471 patients initially identified as hyperkalemia. 248 patients were excluded because of suspected or confirmed hemolysis, leaving 223 cases for analysis. 64 unmatched controls were randomly selected from those with confirmed hemolysis (Table 1). The total follow-up time for all patients was 490 person-years (178850 person days).

Baseline characteristics of study participants are summarized according to case or control status in Table 1. The two groups were similar with respect to gender, age and race distributions. 
Hyperkalemic subjects were statistically significantly more likely to have diabetes mellitus and hypertension, and taking $\beta$-blockers and diuretics. Mean eGFR was significantly lower in the hyperkalemic patients, as was incidence of ESRD. Unadjusted mortality rate in the hyperkalemia group was $46.6 \%$ (104/223, or 27.9 per 100 person-days, $95 \% \mathrm{Cl}$ : 22.8-33.8 per 100 persondays), versus $18.8 \%$ (12/64, 10.2 per 100 person days, $95 \% \mathrm{Cl}$ : 5.3-17.8 per 100 person-days) in controls. Table 2 shows baseline characteristics stratified by serum potassium levels (3.3-5.2, 5.3$6,>6.1 \mathrm{mEq} / \mathrm{L}$ ). Overall, $36.0 \%$ of ESRD patients had a recurrent serum potassium value $\geq 5.3 \mathrm{mEq} / \mathrm{L}$ after the index episode compared to $12.4 \%$ of non-ESRD patients $(p=0.006)$. There were $84(37.7 \%)$ deaths among cases and 16 (25\%) among controls. Figure 1 depicts the Kaplan-Meier curves for cases versus controls, with overall survival probabilities for cases of $50.2 \%$ and $76.5 \%$ in controls (log-rank p-value $=0.001$ ). Of the 64 cases,
44 met our definition of cardiac death, and an additional 2 listed hyperkalemia as the cause of death. Of controls, 3 deaths were coded as cardiac, and none were listed as having hyperkalemia as the cause of death. Hyperkalemic and cardiac death occurred more frequently in cases than controls (odds ratio $=5.2,95 \%$ $\mathrm{Cl}=1.39-19.8, \mathrm{p}=0.01)$. Figure 2 shows the increased risk of death with increasing potassium category based on potassium level at admission.

A positive linear trend in hazards ratios for mortality was observed between potassium level and $\mathrm{CCl}(\mathrm{p}=0.07)$ (Table 1, online supplement). Statistically significant negative linear trend was observed with eGFR ( $p=0.001$ ) (Table 3).

Three hierarchical Cox proportional hazard regression models are displayed in Table 4. The top panel shows analysis of mortality, the lower panel the results of one-year landmark analysis

Table 1 Baseline Characteristics of participants at admission ( $n=287)$.

\begin{tabular}{|c|c|c|c|}
\hline Characteristics* & Cases ( $n=223)$ & Controls $(n=64)$ & p-value ** \\
\hline Gender (Male) & 138 (61.9) & $35(54.7)$ & 0.302 \\
\hline Race (White) & $160(71.7)$ & $49(76.6)$ & 0.9228 \\
\hline Age (Years) & $65.0 \pm 18.0$ & $62.3 \pm 17.8$ & 0.2774 \\
\hline \multicolumn{4}{|c|}{ Renal function } \\
\hline Normal $^{1}$ & $56(25.1)$ & $46(71.9)$ & \multirow{4}{*}{$<0.0001$} \\
\hline Chronic kidney disease stage $3^{2}$ & $67(30.0)$ & $13(20.3)$ & \\
\hline Chronic kidney disease stage $4^{3}$ & $40(17.9)$ & $5(7.8)$ & \\
\hline Kidney failure/Dialysis ${ }^{4}$ & $58(26.0)$ & $0(0.0)$ & \\
\hline End Stage Renal Disease (Dialysis Dependent) & 31 (13.9) & $1(1.6)$ & 0.0009 \\
\hline \multicolumn{4}{|c|}{ Potassium } \\
\hline $3.3-5.2$ & $0(0.0)$ & $64(100.0)$ & \multirow{3}{*}{$<0.0001$} \\
\hline $5.3-6.0$ & $160(71.7)$ & $0(0.0)$ & \\
\hline $6.1+$ & $63(28.3)$ & $0(0.0)$ & \\
\hline Diabetes mellitus & $106(47.5)$ & $21(32.8)$ & 0.0402 \\
\hline Hypertension & $151(67.7)$ & $32(50.0)$ & 0.0186 \\
\hline Congestive heart failure & $66(29.6)$ & $12(18.8)$ & 0.0778 \\
\hline ACE inhibitors or ARB use & $95(42.6)$ & $20(31.3)$ & 0.1183 \\
\hline Beta blockers use & $128(57.4)$ & $27(42.2)$ & 0.0412 \\
\hline NSAIDs use & $21(9.4)$ & $6(9.4)$ & 0.5665 \\
\hline Diuretics use & $94(42.2)$ & $15(23.4)$ & 0.0067 \\
\hline Insulin use & $61(27.4)$ & $8(12.5)$ & 0.0665 \\
\hline Oral hypoglycemic agents use & $28(12.6)$ & $8(12.5)$ & 0.6251 \\
\hline Potassium supplements use & $14(6.3)$ & $1(1.6)$ & 0.0974 \\
\hline MDRD GFR $\left(\mathrm{mL} / \mathrm{min} / 1.73 \mathrm{~m}^{2}\right)$ & $42.4 \pm 35.2$ & $83.5 \pm 34.5$ & $<0.0001$ \\
\hline Potassium (mEq/L) & $5.9 \pm 0.6$ & $4.3 \pm 0.4$ & $<0.0001$ \\
\hline Charlson co-morbidity index (unadjusted), (median, IQR) & $3.0 \pm 3.0$ & $2.0 \pm 3.5$ & 0.001 \\
\hline Systolic blood pressure $(\mathrm{mmHg})$ at Admission & $124.6 \pm 28.1$ & $135.0 \pm 25.8$ & 0.1167 \\
\hline Diastolic blood pressure $(\mathrm{mmHg})$ at Admission & $65.8 \pm 18.8$ & $71.4 \pm 17.5$ & 0.2084 \\
\hline Mean arterial pressure $(\mathrm{mmHg})$ at Admission & $85.4 \pm 19.9$ & $92.6 \pm 16.1$ & 0.1222 \\
\hline
\end{tabular}

*Values are $n(\%)$ for categorical variables and mean \pm standard deviation for continuous variables, unless otherwise noted.

**Two-sample t-test or chi-squared test. ${ }^{1}$ eGFR>60, ${ }^{2}$ eGFR 30-59, ${ }^{3}$ eGFR 15-29, ${ }^{4}$ eGFR 0-14 mL/min/1.73 m² 
Table 2 Baseline characteristics of patients ( $n=287)$ according to admission potassium levels ( $m E q / L)$.

\begin{tabular}{|c|c|c|c|c|}
\hline Characteristics $^{*}$ & $3.3-5.2(n=64)$ & $5.3-6.0(n=160)$ & $6.1+(n=63)$ & Trend $p$-value ${ }^{* *}$ \\
\hline Gender (Male) & $35(54.7)$ & $95(59.4)$ & $43(68.3)$ & 0.2725 \\
\hline Race (White) & $49(76.6)$ & $116(72.5)$ & $44(69.8)$ & 0.9705 \\
\hline Age (Years) & $62.3 \pm 17.8$ & $66.8 \pm 17.6$ & $60.6 \pm 18.2$ & 0.923 \\
\hline \multicolumn{5}{|c|}{ Renal function } \\
\hline Normal $^{1}$ & 46 (71.9) & $48(30.0)$ & $8(12.7)$ & $<0.0001$ \\
\hline Chronic kidney disease stage $3^{2}$ & $13(20.3)$ & $49(30.6)$ & $18(28.6)$ & \\
\hline Chronic kidney disease stage $4^{3}$ & $5(7.8)$ & $28(17.5)$ & $12(19.0)$ & \\
\hline Kidney failure/Dialysis ${ }^{4}$ & $0(0.0)$ & $33(20.6)$ & $25(39.7)$ & \\
\hline End stage renal disease (Dialysis dependent) & $1(1.6)$ & $17(10.6)$ & $14(22.2)$ & 0.0003 \\
\hline Diabetes mellitus & $21(32.8)$ & $73(45.6)$ & $33(52.4)$ & 0.0727 \\
\hline Hypertension & $32(50.0)$ & $109(68.1)$ & $42(66.7)$ & 0.0627 \\
\hline Congestive heart failure & $12(18.8)$ & $49(30.6)$ & $17(27.0)$ & 0.1838 \\
\hline ACE inhibitors or ARB use & $20(31.3)$ & $64(40.0)$ & $31(49.2)$ & 0.1089 \\
\hline Beta blockers use & $27(42.2)$ & $93(58.1)$ & $35(55.6)$ & 0.123 \\
\hline NSAIDs use & $6(9.4)$ & $15(9.4)$ & $6(9.5)$ & 0.8351 \\
\hline Diuretics use & $15(23.4)$ & $69(43.1)$ & $25(39.7)$ & 0.0239 \\
\hline Insulin use & $8(12.5)$ & $43(26.9)$ & $18(28.6)$ & 0.1667 \\
\hline Oral hypoglycemic agents use & $8(12.5)$ & $22(13.8)$ & $6(9.5)$ & 0.6572 \\
\hline Potassium supplements use & $1(1.6)$ & $8(5.0)$ & $6(9.5)$ & 0.1151 \\
\hline MDRD GFR $\left(\mathrm{mL} / \mathrm{min} / 1.73 \mathrm{~m}^{2}\right)$ & $83.5 \pm 34.5$ & $46.6 \pm 34.9$ & $31.9 \pm 34.1$ & $<0.0001$ \\
\hline Charlson co-morbidity index (unadjusted), (median, IQR) & $2.0 \pm 3.5$ & $3.0 \pm 3.0$ & $3.0 \pm 3.0$ & 0.0009 \\
\hline Systolic blood pressure $(\mathrm{mmHg})$ at admission & $135.0 \pm 25.8$ & $126.3 \pm 27.6$ & $120.8 \pm 29.1$ & 0.1721 \\
\hline Diastolic blood pressure $(\mathrm{mmHg})$ at admission & $71.4 \pm 17.5$ & $66.6 \pm 18.2$ & $63.8 \pm 20.2$ & 0.137 \\
\hline Mean arterial pressure $(\mathrm{mmHg})$ at admission & $92.6 \pm 16.1$ & $86.5 \pm 19.5$ & $82.8 \pm 20.8$ & 0.1125 \\
\hline
\end{tabular}

*Values are $n(\%)$ for categorical variables and mean \pm standard deviation for continuous variables, unless otherwise noted.

${ }^{* *}$ Linear regression for continuous traits and Chi-squared test for binary traits. ${ }^{1}$ eGFR>60, ${ }^{2}$ eGFR 30-59, ${ }^{3}$ eGFR $15-29,{ }^{4}$ eGFR 0-14 ml/min/1.73 $\mathrm{m}^{2}$

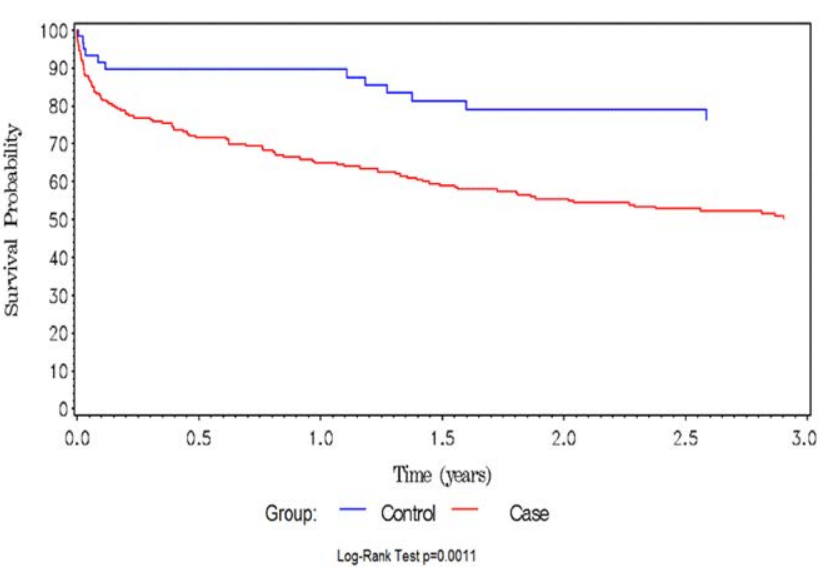

Figure 1 Kaplan-meier plot of time to death cases vs. controls.

restricted to 1-year follow-up. Model 1 was age and sex only adjusted; Model 2 was adjusted for the covariates that attained statistical significance in age and sex adjusted model; and Model 3 was additionally adjusted for $\mathrm{CCl}$. The HRs and 95\% $\mathrm{Cls}$ shown in Table 4 represent changes in HRs for every unit increase in raw potassium, log-potassium, and per 1-SD increase in log potassium, respectively. For each 1 -unit $(1 \mathrm{mEq} / \mathrm{L})$ increase in potassium, the HR for mortality increased by 54\% (HR: 1.54, 95\% $\mathrm{Cl}: 1.22-1.96)$. The HRs and respective c-statistic were unchanged upon additional adjustment for $\mathrm{CCl}$ or upon log-transforming the

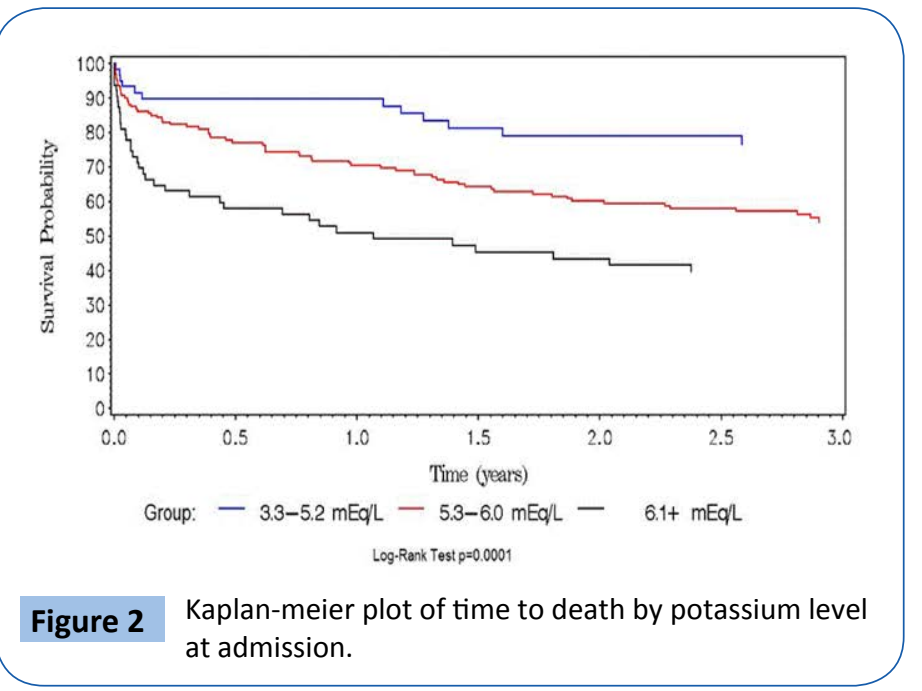

potassium concentration. Compared to the lowest quartile of potassium, the second quartile had nearly twice the hazard for mortality (HR: $1.94,95 \% \mathrm{Cl}: 1.03-3.64)$ and the $4^{\text {th }}$ quartile nearly 3.5 times the hazard (HR: $3.48,95 \% \mathrm{Cl}: 1.78-6.81$ ), therefore suggesting a nonlinear relationship with increasing potassium levels. The non-linear relationship of mortality hazard with increasing potassium concentrations was examined using cubic splines (Figure 3, online supplement).

The HRs for respective potassium quartiles were qualitatively unchanged upon additional adjustment for $\mathrm{CCl}$ severity. In the 
Table 3 Cox proportional hazard models examining the relations of MDRD-GFR $\left(\mathrm{mL} / \mathrm{min} / 1.73 \mathrm{~m}^{2}\right)$ to mortality.

\begin{tabular}{|c|c|c|c|c|}
\hline \multirow{2}{*}{ Model } & Model $1^{*}$ & \multirow[b]{2}{*}{ P value } & Model $2^{+}$ & \\
\hline & Hazard Ratio (95\% Cl) & & Hazard Ratio $(95 \% \mathrm{Cl})$ & $P$ value \\
\hline \multicolumn{5}{|c|}{ Overall Survival $^{1}$} \\
\hline MDRD GFR at Baseline & $0.99(0.98-0.99)$ & 0.0001 & $0.99(0.99-1.00)$ & 0.0076 \\
\hline Log-transformed MDRD GFR, unit increment & $0.70(0.55-0.87)$ & 0.0019 & $0.75(0.59-0.95)$ & 0.0164 \\
\hline $\begin{array}{l}\text { Standardized log-transformed MDRD GFR, 1-SD } \\
\text { increment }\end{array}$ & $0.73(0.60-0.89)$ & 0.0019 & $0.77(0.63-0.95)$ & 0.0164 \\
\hline \multicolumn{5}{|c|}{ Quartiles of MDRD GFR ${ }^{* *}$} \\
\hline Quartile 1 & \multicolumn{2}{|c|}{ Referent } & \multicolumn{2}{|l|}{ Referent } \\
\hline Quartile 2 & $0.64(0.41-1.02)$ & 0.0592 & $0.75(0.46-1.21)$ & 0.2364 \\
\hline Quartile 3 & $0.43(0.26-0.70)$ & 0.0007 & $0.59(0.35-0.99)$ & 0.044 \\
\hline Quartile 4 & $0.25(0.13-0.48)$ & $<0.0001$ & $0.35(0.18-0.69)$ & 0.0024 \\
\hline Trend across quartiles & & $<0.0001$ & & 0.0012 \\
\hline \multicolumn{5}{|l|}{ 1-year Survival ${ }^{2}$} \\
\hline MDRD GFR at Baseline & $0.99(0.98-0.99)$ & 0.0002 & $0.99(0.98-1.00)$ & 0.0101 \\
\hline Log-transformed MDRD GFR, unit increment & $0.64(0.49-0.83)$ & 0.0009 & $0.68(0.52-0.90)$ & 0.0061 \\
\hline $\begin{array}{l}\text { Standardized log-transformed MDRD GFR, 1-SD } \\
\text { increment }\end{array}$ & $0.68(0.54-0.85)$ & 0.0009 & $0.72(0.57-0.91)$ & 0.0061 \\
\hline \multicolumn{5}{|c|}{ Quartiles of MDRD GFR ${ }^{* *}$} \\
\hline Quartile 1 & \multicolumn{2}{|c|}{ Referent } & \multicolumn{2}{|l|}{ Referent } \\
\hline Quartile 2 & $0.73(0.43-1.25)$ & 0.251 & $0.84(0.48-1.47)$ & 0.54 \\
\hline Quartile 3 & $0.39(0.21-0.72)$ & 0.0026 & $0.59(0.31-1.11)$ & 0.1025 \\
\hline Quartile 4 & $0.24(0.10-0.55)$ & 0.0008 & $0.33(0.14-0.80)$ & 0.0134 \\
\hline Trend across quartiles & & $<0.0001$ & & 0.0066 \\
\hline
\end{tabular}

"Models adjusted for baseline age and sex; ${ }^{\dagger}$ Models adjusted for baseline age, sex, and significant covariates from univariate models (history of congestive heart failure and use of oral hypoglycemic agents); ${ }^{1}$ Overall deaths=116; 21-year deaths=82; ${ }^{* *}$ Quartile $1=0-17.2\left(\mathrm{~mL} / \mathrm{min} / 1.73 \mathrm{~m}{ }^{2}\right.$; Quartile 2=17.3-42.1 (mL/min/1.73 $\left.\mathrm{m}^{2}\right)$; Quartile 3=43.0-80.2 (mL/min/1.73 $\left.\mathrm{m}^{2}\right)$; Quartile 4=80.6-140.0 (mL/min $\left./ 1.73 \mathrm{~m}^{2}\right)$

landmark analysis, the same pattern that was observed in the overall analysis emerged even though the HRs were moderately higher. The HR associated with each 1-unit increase in potassium was 1.83 (95\% Cl: 1.38-2.44) (Table 4). The HRs for parallel analyses using $\mathrm{CCl}$ as the exposure variable instead of potassium concentration (Table 1, online supplement) reveal that $\mathrm{CCl}$ was significantly associated with mortality in age and sex only adjusted models; in the overall analysis the associations become somewhat attenuated upon additional adjustment and did not attain statistical significance in the landmark analysis. Each 1-unit increase in $\mathrm{CCl}$ increased the hazard for mortality by $10 \%$ (HR: 1.10, 95\% Cl: 1.02-1.18).

The HRs using MDRD-GFR as the exposure variable (Table 3) revealed a significant protective effect; for each 1-unit increase in MDRD-GFR, the hazard for mortality decreases by $1 \%$. Similar results were observed in the landmark analysis. The protective effect of MDRD-GFR was more pronounced in the third and fourth quartiles relative to the first quartile (HR: $0.59,95 \% \mathrm{Cl}$ : $0.35-0.99$ for $3^{\text {rd }}$ quartile: $0.35,95 \% \mathrm{Cl}: 0.18-0.69$ in the highest quartile). Kaplan-Meier curves showed that overall survival probability declined with decreasing MDRD-GFR at admission (log-rank p-value<0.0001) (Figure 2, online supplement). The non-linear pattern of the HRs with increasing MDRD-GFR was examined using cubic splines (Figure 4, online supplement).

Excess 1-year mortality was seen in the HF patients with hyperkalemia $(58.3 \%$ versus $17.5 \%$ in controls with HF, log-rank $\mathrm{p}$-value $=0.03$ ). Overall survival probability differed significantly between HF patients and those with no history of HF (log-rank p-value<0.0001) (Figure 4).

To explore the joint relationship of potassium and MDRD-GFR, we used the composite outcome based on the $80^{\text {th }}$ percentile of admit potassium $(6.1 \mathrm{mEq} / \mathrm{L})$ and $20^{\text {th }}$ percentile of MDRD-GFR $(15.11 \mathrm{ml} / \mathrm{min})$. The Kaplan-Meier plots of the four composite categories showed a statistically significant difference (log-rank test $\mathrm{p}$-value<0.0001) (Figure 1, online supplement). The HR for the High K/Low GFR group was 3.75 (95\% Cl: 2.20-6.41). Crude mortality rate was the highest in the High K/Low GFR category [80.6\% (Cl 61.9-93.8)], intermediate in High K/High GFR category [43.0\% (Cl 27.0-63.3)], and the Low K/Low GFR [59.0\% (Cl 42.875.8)], and lowest in Low K/High GFR category [38.4\% (Cl 31.446.3)].

Kaplan-Meier survival curves showed statistically significantly better survival in ESRD patients compared to severe AKI patients (log-rank p-value $=0.04$ ). Overall survival in ESRD patients was $37.6 \%$ compared to severe AKI group $22.5 \%$ ( $n=32$ ESRD patients vs. $n=15$ severe AKI patients). Mortality rate was $68.8 \%$ in AKI patients vs. $43.2 \%$ in non-AKI patients for overall follow-up time (log-rank $p=0.01$ ) (Figure 5, online supplement).

\section{Discussion}

There are no intermediate and long-term mortality studies on the impact of single episode of hyperkalemia in the ED or general population. The current literature focuses largely on acute 
Table 4 Cox proportional hazard models examining the relations of potassium levels $(\mathrm{mEq} / \mathrm{L})$ to mortality.

\begin{tabular}{|c|c|c|c|c|c|c|c|c|c|}
\hline \multirow[t]{2}{*}{ Model } & \multicolumn{3}{|l|}{ Model $1^{*}$} & \multicolumn{3}{|c|}{ Model $2^{+}$} & \multicolumn{3}{|c|}{ Model $3^{\ddagger}$} \\
\hline & Hazard Ratio (95\% Cl) & P value & C & Hazard Ratio $(95 \% \mathrm{Cl})$ & $P$ value & C & Hazard Ratio (95\% Cl) & P value & C \\
\hline \multicolumn{10}{|c|}{ Overall Survival $^{1}$} \\
\hline $\begin{array}{l}\text { Potassium at } \\
\text { Baseline }\end{array}$ & $1.67(1.34-2.08)$ & $<0.0001$ & 0.7 & $1.54(1.22-1.96)$ & 0.0003 & 0.71 & $1.55(1.22-1.96)$ & 0.0004 & 0.72 \\
\hline $\begin{array}{l}\text { Log-transformed } \\
\text { potassium, unit } \\
\text { increment }\end{array}$ & 20.35 (5.26-78.75) & $<0.0001$ & 0.7 & $12.90(3.12-53.38)$ & 0.0004 & 0.71 & $12.85(3.06-53.96)$ & 0.0005 & 0.72 \\
\hline $\begin{array}{l}\text { Standardized } \\
\text { Log-transformed } \\
\text { potassium, 1-SD } \\
\text { increment }\end{array}$ & $1.66(1.32-2.08)$ & $<0.0001$ & 0.7 & $1.54(1.21-1.95)$ & 0.0004 & 0.71 & $1.53(1.21-1.95)$ & 0.0005 & 0.72 \\
\hline \multicolumn{10}{|c|}{ Quartiles of Potassium ${ }^{* *}$} \\
\hline Quartile 1 & \multicolumn{3}{|l|}{ Referent } & \multicolumn{3}{|c|}{ Referent } & \multicolumn{3}{|c|}{ Referent } \\
\hline Quartile 2 & $1.99(1.07-3.71)$ & 0.0304 & 0.69 & $1.94(1.03-3.64)$ & 0.0389 & 0.71 & $1.91(1.02-3.60)$ & 0.0443 & 0.71 \\
\hline Quartile 3 & $1.56(0.79-3.07)$ & 0.2017 & & $1.44(0.72-2.87)$ & 0.3052 & & $1.44(0.72-2.87)$ & 0.3047 & \\
\hline Quartile 4 & $3.82(2.03-7.22)$ & $<0.0001$ & & $3.48(1.78-6.81)$ & 0.0003 & & $3.38(1.72-6.63)$ & 0.0004 & \\
\hline Trend a & cross quartiles & 0.0001 & & & 0.0019 & & & 0.0027 & \\
\hline \multicolumn{10}{|c|}{ 1-year Survival ${ }^{2}$} \\
\hline $\begin{array}{c}\text { Potassium at } \\
\text { Baseline }\end{array}$ & $1.90(1.46-2.48)$ & $<0.0001$ & & $1.83(1.38-2.44)$ & $<0.0001$ & & $1.82(1.36-2.43)$ & $<0.0001$ & \\
\hline $\begin{array}{l}\text { Log-transformed } \\
\text { potassium, unit } \\
\text { increment }\end{array}$ & $49.51(9.46-259.13)$ & $<0.0001$ & & 38.09 (6.47-224.19) & $<0.0001$ & & $36.43(6.13-216.61)$ & $<0.0001$ & \\
\hline $\begin{array}{l}\text { Standardized } \\
\text { Log-transformed } \\
\text { potassium, 1-SD } \\
\text { increment }\end{array}$ & $1.92(1.46-2.54)$ & $<0.0001$ & & $1.84(1.37-2.48)$ & $<0.0001$ & & $1.83(1.36-2.46)$ & $<0.0001$ & \\
\hline \multicolumn{10}{|c|}{ Quartiles of Potassium ${ }^{* *}$} \\
\hline Quartile 1 & Referent & & & Referent & & & Referent & & \\
\hline Quartile 2 & $2.90(1.20-6.99)$ & 0.018 & & $2.94(1.22-7.11)$ & 0.0168 & & $2.97(1.22-7.19)$ & 0.0161 & \\
\hline Quartile 3 & $2.29(0.90-5.83)$ & 0.0834 & & $2.13(0.83-5.47)$ & 0.1169 & & $2.11(0.82-5.41)$ & 0.1224 & \\
\hline Quartile 4 & $6.64(2.76-15.97)$ & $<0.0001$ & & $6.58(2.65-16.30)$ & $<0.0001$ & & $6.31(2.54-15.71)$ & $<0.0001$ & \\
\hline \multicolumn{2}{|c|}{ Trend across quartiles } & $<0.0001$ & & & 0.0001 & & & 0.0003 & \\
\hline
\end{tabular}

*Models adjusted for baseline age and sex; ${ }^{+}$Models adjusted for baseline age, sex, and significant covariates from univariate models (history of end stage renal disease, history of congestive heart failure); ${ }^{\ddagger}$ Models adjusted for all covariates as in model 2 and additionally for unadjusted Charlson Score; ${ }^{1}$ Overall deaths $=116 ;{ }^{2} 1$-year deaths $=82 ;{ }^{* *}$ Quartile 1=3.3-5.3 (mEq/L); Quartile 2=5.4-5.6 (mEq/L); Quartile 3=5.7-6.0 (mEq/L); Quartile $4=6.1-8.3(\mathrm{mEq} / \mathrm{L})$

mortality of hyperkalemia. Cross-sectional analyses using large databases have shown increased mortality with hyperkalemia $[1,20]$. Few studies on hospitalized hyperkalemic patients studied short term mortality [21]. To our knowledge, there is no longitudinal study or follow-up studies on ED hyperkalemic patients. Furthermore, the predictors for long term mortality in survivors from index hyperkalemia have not been studied. Identification of these predictors will help to target high risk mortality groups with novel therapeutic agents $[5,6]$.

In our study, the deleterious effect of admit hyperkalemia on allcause mortality even after discharge from index ED hyperkalemia was evident. More than 3 out of 4 deaths in cases occurred after discharge from index ED episode. This effect was especially marked in cases with HF who had a 58.3\% 1-year mortality rate compared to $17.5 \%$ in controls with $\mathrm{HF}$, in contrast to the expected 1-year mortality (30-40\%) in HF patients in USA surviving hospitalization [22].
The hazard ratios for mortality associated with admit potassium were higher, compared to the hazards associated with validated $\mathrm{CCl}$. Renal function as estimated by MDRD GFR at admit, had significant protective effect on mortality. The dynamic changes in renal function as studied by AKI and severe AKI subsets had a greater role on all-cause mortality compared to static value of admit eGFR. Interestingly ESRD patients had better survival compared to severe AKI, perhaps owing to the effect of physiologic adaptations to repeated episodes of hyperkalemia [23].

Hyperkalemia plays a significant role in certain CV disease [24] and is major factor affecting 30 day mortality and ventricular arrhythmias in Acute Myocardial Infarction [25] with a U-shaped relation of serum potassium with short term mortality/ arrhythmias especially with serum potassium of above $4.5 \mathrm{mEq} / \mathrm{L}$ [25]. This finding substantiates our hypothesis that myocardium may be more susceptible to hyperkalemia, especially in acute myocardial infarction and possibly in HF. 
In the context of acute myocardial infarction, two studies shed light on long term mortality implications of serum potassium levels $>4.5 \mathrm{mEq} / \mathrm{L}$. Shiyovich et al. showed that potassium $>4.5$ $\mathrm{mEq} / \mathrm{L}$ is significantly associated with increased post-discharge long-term mortality in acute $\mathrm{Ml}$ over median follow up of 8.1 years [26]. A similar study Choi et al showed mortality risk was greater for serum potassium $>4.5 \mathrm{mEq} / \mathrm{L}$ (HR1.71, 95\% [Cl] 1.04 to 2.814 .5 to $<5.0 \mathrm{mEq} / \mathrm{L}$ and $\mathrm{HR} 4.78,95 \% \mathrm{Cl} 2.14$ to 10.69 for $>5.0$ when compared to patients with potassium levels of 3.5 to $<4.0 \mathrm{mEq} / \mathrm{L}$ over mean of 3.58 year follow-up [27]

These two studies however include acute myocardial infraction subjects only and not applicable to general population. Our study is the only one to our knowledge which studied long term outcomes of single episode of hyperkalemia in a population of heterogeneous subject who presented to Emergency department and likely representative of general population.

A few studies on the CKD population have showed a $U$ shaped relationship between serum potassium and mortality. Serum potassium $>5.0 \mathrm{mmol} / \mathrm{L}$ was associated with increased mortality in non-dialysis dependent CKD patients [28]. A secondary analysis of the Renal Research Institute study CKD cohort also showed an increased risk of the composite of death or cardiovascular events requiring hospitalization but no increase in overall mortality with serum potassium $>5.5 \mathrm{mmol} / \mathrm{L}$ [29]. These studies are limited to CKD subjects only.

The mortality observed in the HF subset may be attributed to cardiac arrhythmias [30]. Hyperkalemia may be a surrogate marker for mortality or decompensated HF. Our findings are in contrast with short term data from inpatients (21) where HF was not a major predictor of short term mortality. The myocardium in advanced CKD non ESRD patients may be more sensitive to arrhythmogenic effects of hyperkalemia secondary to increased left ventricular mass or scarring. Current literature points to low GFR or reduced renal function as an independent risk factor for cardiac arrhythmias in varied populations $[30,31]$ and increased mortality among patients with implantable cardiac defibrillators [32]. This interaction between low GFR and HF in the hyperkalemic patient needs to be further explored. Renal function is a risk factor for increased mortality in HF patients $[33,34]$ and fatal cardiovascular disease in older patients [35].

Our study findings show concurrence with the findings of Jain et al. [18] on patients with cardiovascular disease (defined in the study as hypertension and heart failure) and associated CKD where predictors of all-cause mortality included CKD stage (odds ratio [OR]: 1.26) and hyperkalemic event (>5 mEq/I) [OR: 1.56]. but follow up period was not specified.

Even mild hyperkalemia may predispose cardiovascular disease patients with impaired renal function to higher mortality as shown in our study. These data suggest that patients with hyperkalemia and concurrent $\mathrm{HF}, \mathrm{AKI}$, or low admit eGFR may need close monitoring and these patients need to be identified as high risk patients for interventions to prevent or treat hyperkalemia, especially after discharge from ED.

The persistence of the mortality risk after hyperkalemic episode is the high light of our study and calls for closer monitoring and intervention possibly with newer therapies in the followup period. Future studies are needed to explore the mortality benefits of new hyperkalemia therapies $[5,6]$.

\section{Limitations}

Our study is inherently limited by its retrospective, single center design and that the cases were not individually matched to the controls which may cause residual confounding. Our study excluded patients with confirmed hemolysis (pseudohyperkalemia), and controls were derived from that population. Limited data on hemolysis incidence is available in the exiting literature. We paid meticulous attention to hemolysis maintaining the separation between cases and controls throughout the study period and up to 6 months later. Although our analysis has the significant advantage of being a clean database for studying true hyperkalemia, local variation in the accuracy of potassium measurements may limit the generalization of these results. The findings pertain to ED patients and may not be applicable to the general population.

\section{Conclusions}

Hyperkalemia was a strong predictor for all-cause mortality especially over the longer term, in the heterogeneous ED patient population even when adjusted for age, sex, Charlson comorbidity index, and significant covariates on univariate analysis, HF and ESRD. Excess hazards for all-cause mortality persisted thought out the follow up study period of 490 patient years in survivors from the index episode. Cardiovascular mortality was statistically more prevalent in hyperkalemic patients. Initial hyperkalemia was a stronger predictor for all-cause mortality than Charlson comorbidity index. Admit GFR had a protective non-linear effect on mortality. HF patients with hyperkalemia had higher risk of mortality.

The persistence of the mortality risk after hyperkalemic episode in our study calls for evolution of risk stratification strategies for hyperkalemia on the longer term, need for future studies to evaluate the mortality benefits of new hyperkalemia therapies [6] so as to evolve strategies that benefit higher risk mortality patients. Our study is a vital step in the identification of high risk mortality patients and evaluate the longer term mortality implications of hyperkalemia.

\section{Acknowledgements}

The authors thank Dr. L Michael Snyder MD, Professor of Pathology, University of Massachusetts Medical School, Dr. L.V. Rao Ph.D., Clinical Associate Professor of Pathology, University of Massachusetts Medical School and Dr. Bruce A. Barton Ph.D., M.S. (Hyg.), M.A. Research Professor, Department of Quantitative Health Science for their support.

\section{Funding}

Aimee and Dr. P Gona were supported by University of Massachusetts CTSA grant number UL1RR031982. 


\section{References}

1 Einhorn LM, Zhan M, Hsu VD, Walker LD, Moen MF, et al. (2009) The frequency of hyperkalemia and its significance in chronic kidney disease. Arch Intern Med 169: 1156-1162.

2 Hemels ME, Bennett HA, Bonari L, Han D, Traverso ML, et al. (2003) HOPE study impact on ACE inhibitors use. Ann Pharmacother 37: 640-645.

3 Incalzi AR, Pedone C, Pahor M, Onder G, Carbonin PU (2002) Trends in prescribing $A C E$-inhibitors for congestive heart failure in elderly people. Aging Clin Exp Res. 14: 516-521.

4 Bakris GL, Siomos M, Richardson D, Janssen I, Bolton WK, et al. (2000) ACE inhibition or angiotensin receptor blockade: Impact on potassium in renal failure. VAL-K Study Group. Kidney Int 58: 2084-2092.

5 Packham DK, Rasmussen HS, Lavin PT, El-Shahawy MA, Roger SD, et al. (2015) Sodium zirconium cyclosilicate in hyperkalemia. N Engl J Med 372: 222-231.

6 Weir MR, Bakris GL, Bushinsky DA, Mayo MR, Garza D, et al. (2015) Patiromer in patients with kidney disease and hyperkalemia receiving RAAS inhibitors. N Engl J Med 372: 211-221.

7 Desai AS, Swedberg K, McMurray JJ, Granger CB, Yusuf S, et al. (2007) Incidence and predictors of hyperkalemia in patients with heart failure: an analysis of the CHARM Program. J Am Coll Cardiol 50: 1959-1966.

8 Wentworth DN, Neaton JD, Rasmussen WL (1983) An evaluation of the Social Security Administration master beneficiary record file and the National Death Index in the ascertainment of vital status. Am J Public Health 73: 1270-1274.

9 Tseng ZH, Secemsky EA, Dowdy D, Vittinghoff E, Moyers B, et al. (2012) Sudden cardiac death in patients with human immunodeficiency virus infection. J Am Coll Cardiol 59: 1891-1896.

10 Chung CP, Murray KT, Stein CM, Hall K, Ray WA (2010) A computer case definition for sudden cardiac death. Pharmacoepidemiol Drug Saf 19: 563-572.

11 Levey AS, Coresh J, Greene T, Stevens LA, Zhang YL, et al. (2006) Using standardized serum creatinine values in the modification of diet in renal disease study equation for estimating glomerular filtration rate. Ann Intern Med 145: 247-254.

12 Charlson ME, Pompei P, Ales KL, MacKenzie CR (1987) A new method of classifying prognostic comorbidity in longitudinal studies: Development and validation. J Chronic Dis 40: 373-383.

13 Christensen S, Johansen MB, Christiansen CF, Jensen R, Lemeshow $S$ (2011) Comparison of Charlson comorbidity index with SAPS and APACHE scores for prediction of mortality following intensive care. Clin Epidemiol 3: 203-211.

14 Bellomo R, Ronco C, Kellum JA, Mehta RL, Palevsky P (2004) Acute renal failure-definition, outcome measures, animal models, fluid therapy and information technology needs: The Second International Consensus Conference of the Acute Dialysis Quality Initiative (ADQI) Group. Crit Care 8: R204-R212.

15 Cox D (1972) Regression models and life tables. J Roy Statis Society $34: 187-220$

16 Pencina MJ, D'Agostino RB (2004) Overall C as a measure of discrimination in survival analysis: model specific population value and confidence interval estimation. Stat Med 23: 2109-2123.

17 Desquilbet L, Mariotti F (2010) Dose-response analyses using restricted cubic spline functions in public health research. Statistics Med 29: 1037-1057.
18 Greenland S (1995) Dose-response and trend analysis in epidemiology: alternatives to categorical analysis. Epidemiology 6: 356-365.

19 Hastie T, Tibshirani R (1995) Generalized additive models for medical research. Stat Methods Med Res 4: 187-196.

20 Jain N, Kotla S, Little BB, Weideman RA, Brilakis ES, et al. (2012) Predictors of hyperkalemia and death in patients with cardiac and renal disease. Am J Cardiol 109: 1510-1513.

21 An JN, Lee JP, Jeon HJ, Kim DH, Oh YK, et al. (2012) Severe hyperkalemia requiring hospitalization: Predictors of mortality. Crit care 16: R225.

22 Goldberg RJ, Ciampa J, Lessard D, Meyer TE, Spencer FA (2007) Longterm survival after heart failure: A contemporary population-based perspective. Arch Intern Med 167: 490-496.

23 Gennari FJ, Segal AS (2002) Hyperkalemia: An adaptive response in chronic renal insufficiency. Kidney Int 62: 1-9.

24 Goyal A, Spertus JA, Gosch K, Venkitachalam L, Jones PG, et al. (2012) Serum potassium levels and mortality in acute myocardial infarction. JAMA 307: 157-164.

25 Scirica BM, Morrow DA (2012) Potassium concentration and repletion in patients with acute myocardial infarction. JAMA 307: 195-196.

26 Shiyovich A, Gilutz H, Plakht Y (2014) Letter to the Editor. International Journal of Cardiology 2014172: e368-e70.

27 Kim CS, Oak CY, Kim HY, Kang YU, Choi JS, et al. (2013) Incidence, predictive factors, and clinical outcomes of acute kidney injury after gastric surgery for gastric cancer. PLOS ONE 8: e82289.

28 Nakhoul GN, Huang H, Arrigain S, Jolly SE, Schold JD, et al. (2015) Serum potassium, end-stage renal disease and mortality in chronic kidney disease. Am J Nephrol 41: 456-463.

29 Korgaonkar S, Tilea A, Gillespie BW, Kiser M, Eisele G, et al. (2010) Serum potassium and outcomes in CKD: Insights from the RRI-CKD cohort study. Clin J Am Soc Nephrol 5: 762-769.

30 Kreuz J, Horlbeck F, Hoyer F, Mellert F, Fimmers R, et al. (2011) An impaired renal function: A predictor of ventricular arrhythmias and mortality in patients with nonischemic cardiomyopathy and heart failure. Pacing Clin Electrophysiol. 34: 894-899.

31 Alonso A, Agarwal SK, Soliman EZ, Ambrose M, Chamberlain AM, et al. (2009) Incidence of atrial fibrillation in whites and AfricanAmericans: the Atherosclerosis Risk in Communities (ARIC) study. Am Heart J 158: 111-117.

32 Kramer DB, Friedman PA, Kallinen LM, Morrison TB, Crusan DJ, et al (2012) Development and validation of a risk score to predict early mortality in recipients of implantable cardioverter-defibrillators. Heart Rhythm 9: 42-46.

33 Hillege HL, Nitsch D, Pfeffer MA, Swedberg K, McMurray JJ, et al. (2006) Renal function as a predictor of outcome in a broad spectrum of patients with heart failure. Circulation 113: 671-678.

34 Peterson PN, Rumsfeld JS, Liang L, Albert NM, Hernandez AF, et al. (2010) A validated risk score for in-hospital mortality in patients with heart failure from the American Heart Association get with the guidelines program. Circ Cardiovasc Qual Outcomes 3: 25-32.

35 Deo R, Fyr CL, Fried LF, Newman AB, Harris TB, et al. (2008) Kidney dysfunction and fatal cardiovascular disease--an association independent of atherosclerotic events: Results from the Health, aging, and body composition (Health ABC) study. Am Heart J 155: 62-68. 